\title{
Research Dialogue \\ Some insights on visual and verbal processing strategies
}

\author{
Richard P. Bagozzi \\ University of Michigan, USA \\ Available online 25 October 2008
}

\begin{abstract}
After briefly describing Wyer's general conceptual framework, the information system, and Barsalou's related perspective, a summary of many of the contributions made by Wyer, Hung, and Jiang (in press) is done. Next shortcomings of the style of processing scale are mentioned, as this is the primary way that chronic processing styles are implemented in the studies discussed in 'Wyer et al. (in press). Following this, a number of philosophical and neuroscience issues are raised. Finally, the commentary closes with questions concerning the role of visual and verbal processing strategies and styles in persuasive communication, the role of affect and sensory processes in social comprehension and judgment, and the need to consider comprehension, judgment, and other cognitive processes in post-decision phases of goal striving.
\end{abstract}

(C) 2008 Society for Consumer Psychology. Published by Elsevier Inc. All rights reserved.

\section{Some background}

Wyer et al. (in press) present an impressive body of research investigating (1) the role of visual imagery in comprehending verbal information, (2) the roles of visual and verbal information processing strategies in making judgments under certain informational, situational, and individual difference conditions, and (3) the effect of verbal coding of visual information during goal pursuit on information use. To appreciate the contributions of all this research, it is helpful to first briefly discuss Wyer's representation of what he terms, the information-processing system (e.g., Wyer, 2004; Wyer \& Radvansky, 1999; Wyer \& Srull, 1989).

Wyer's framework depicts cognitive operations in a set of specialized processing units. Informational stimuli are first processed by the comprehender which uses declarative knowledge as the basis of interpretation, where declarative knowledge can be either categorical (e.g., broad classes of referents) or referent specific (e.g., particular objects, persons, or situations). Especially relevant for comprehension processes are situational models which depict a person's understanding of particular events or states of affairs. The information in a situation model resides in "spatial-temporal context of events..., the entities involved, and... the various relations among these entities" (Wyer \& Radvansky, 1999, p. 98). Wyer notes that situational models are constructed during language comprehen-

E-mail address: bagozzi@umich.edu. sion and also formed as a result of personal involvement with information (also termed, autobiographical memory). At the same time, situation models can affect comprehension. The operation of the comprehender is claimed to draw on knowledge of particular persons and past experiences to interpret new information, where "accessibility of a representation is a function of only the frequency and recency with which it has been used in the past and the degree to which its contents match a memory cue" (Wyer \& Radvansky, 1999, p.92). Moreover, the comprehender is an automatic operator and functions independently of goal-directed processes.

The output of the comprehender, information comprehended, can be processed further in various routines associated with encoding, organization, inference making, and response selection related to this information. This occurs in working memory and like the comprehender draws upon knowledge in the permanent storage unit (long-term memory). But in this case, procedural knowledge can also be important in social cognition. Procedural knowledge consists of cognitive procedures or productions (which are sometimes organized in schemas). The functioning of these procedures can be under conscious control or automatic. Goal-directed processing is a central activity in post-comprehender processing. The type of knowledge most relevant to specific processing objectives (which Wyer notes is reflected in such instances as forming impressions or making decisions) are generalized representations. Generalized representations are "constructed" during comprehension, but especially in goal striving pursuits, and frequently entail an integration of new information with existing knowledge. 
Wyer notes that comprehension can occur during initial encoding where either comparisons to stored knowledge about events, objects, or persons are used to interpret information or a new model is constructed. This process is believed to occur as follows. In a component of the comprehender termed, the parser, information is transformed into a proposition (for verbal information at least), and further processing occurs in a second component of the comprehender: the constructor. Here either existing situation models are identified or new knowledge is constructed based on the permanent storage unit (Wyer \& Radvansky 1999, p. 101). A probing of cues in memory is done by use of the subject and/or predicate in the proposition formed in the parser. Thus either retrieval or construction processes are done to achieve comprehension. Both, however, are largely automatic in operation. If further, more controlled processing is needed, such as in goal-directed contexts, higher-order cognitive operations may be performed.

As a point of comparison, it is useful to consider the framework proposed by Barsalou. Barsalou (1991) argues that knowledge in the cognitive system is represented in categories, and there are two fundamental ways categories originate: through exemplar learning or conceptual combination. Exemplar learning is central to acquiring taxonomic knowledge about the world as it exists and is a relatively passive, bottomup, and automatic process. Goal-derived categories, in contrast, are believed to arise through conceptual combination by manipulation of existing knowledge in memory. Barsalou (1991, p. 4) summarizes aspects of categorization in this sense as follows: "conceptual combination appears to be relatively active, top-down, and effortful. By deliberately manipulating knowledge through reasoning, people produce new categories that serve their goals...conceptual combination often produces idealized knowledge about how the world should be...rather than...about how it is."

Barsalou (1991, p. 27) further proposes that goals and their attributes are represented in frames, which he defines as "flexible, loosely organized bodies of knowledge". For example, the frame for a vacation goal might consist of the vacation category and its connections to five attributes: locations, temporal parameters, activities, objects, and actors. Each attribute, in turn, might be connected to clusters of more specific attributes; for example, departure, duration, return, and schedule are types of temporal parameters, and preparations, travel, and entertainment are types of activities. The specific attributes further might be composed of subtypes; for example, major travel (flying transcontinentally), minor travel (taxi from airport to hotel), and arrangements at location (reserving a seat on a tour bus) are subtypes of travel. Barsalou (1991) shows how such frames are useful in planning goals and how other goals and constraints promote planning. For example, in planning a vacation, such background goals as "maximize relaxation and educational value" guide the selection of exemplars for a frame instantiation. Within a particular frame, certain attributes also constrain the range of other attributes; for example, a needed departure of July for a snow-skiing vacation requires a ski resort in the southern hemisphere. Much remains to be done in the study of the representation of consumer goals, but recent research and insights in memory for brands and attributes and in responses to ads promise to deepen our understanding of goals and information processing (e.g., Alba, Hutchinson, \& Lynch, 1991; Escalas \& Luce, 2004; Luce, 1998; Schwarz, 2004). Wyer's approach, in comparison to Barsalou's framework, focuses more on comprehension, judgment, and the role of processing strategies and dispositions, than goal-directed processing, per se, at least in the paper under discussion.

With the above as background, it is easier to interpret the contributions made by Wyer et al. (in press). The program of research they present makes contributions to the processing of social information with respect to comprehension and judgment processes, and particularly with respect to visual and verbal strategies and dispositions for processing.

\section{Summary of contributions by Wyer et al. (in press)}

An important and original contribution by Wyer, Adval, and Colcombe (2002) is the demonstration of asymmetries in the coding of visual and verbal event descriptions. Their findings show that people spontaneously encode verbal statements visually (as well as semantically) at the time they read such statements, and later are able to verify the occurrence of the events in the statements equally well for verbal or visual presentation, whereas people who are exposed to pictures initially apparently encode the information only visually and at a later time must encode the visual representation verbally when verifying the content. The processes revealed here may have implications for advertising exposure sequences where people first hear a message (say on the radio) and later are exposed to a print ad or television ad (and vice versa).

A second contribution occurs in the research of Jiang and Wyer (2007). They showed that a long-standing finding in the psychology literature concerning the effects of visual perspectives in the comprehension of verbal description of events can be qualified by audience members' dispositions to process stimuli visually or verbally. People dispositionally prone to visual processing construct visual representations whether the mode of presentation is pictorial or verbal. Such persons construct verbal representation only when the information is presented verbally or they are instructed in a way that requires a verbal representation to attain a goal. In a parallel way, people dispositionally prone to semantic processing construct verbal representations regardless of the mode of presentation, and form visual representations only if a goal they have requires this. Chronic processing strategies thus seem to exist where people show a predilection for visual or verbal processing strategies. One wonders whether there are people who have a tendency or skill to process by both modes and what this implies for persuasive communication.

Still another novel and important contribution in Wyer et al. (in press) is that the presentation of visual information is not always effective and can be detrimental. Jiang, Steinhart, and Wyer (2007) studied the effects of pictures on the processing of verbal information and found consistent differences between visualizers and verbalizers, whether studied as a situational prime or a chronic disposition. Thus for visualizers, the 
evaluation of a familiar product was the same whether presented with verbal attribute descriptions alone or verbal attribute descriptions with an added picture; by contrast, the evaluation of an unfamiliar product by visualizers was unfavorable when provided only with verbal attributes in comparison with the case when both verbal attributes and a picture were presented. The opposite occurred for verbalizers. Here the evaluation of a familiar product when a picture was presented was more favorable than when only verbal attribute descriptions were presented. For verbalizers, the evaluation of an unfamiliar product was actually lower when a picture was added to verbal attribute descriptions. We thus see a case where the presentation of visual information actually interferes with verbal information processing and leads to less favorable evaluations. Their results help us explain findings reported in the study by Unnava and Burnkrant (1991), which cannot be elaborated on here in the interest of brevity.

Jiang et al. (2007) also demonstrated the effect of image incompatibility on information processing. An interesting case occurs when visual and verbal aspects of a communication refer to different product attributes. Jiang et al. found that verbalizers encoded visual aspects semantically and combined these with verbally described attributes. However, visualizers found it more difficult to construct a visual image of attributes presented verbally, which hampered the integration of this information with visual presentation of companion attributes. Verbalizers thus evaluated the product more favorably than visualizers. One question for future study is to ascertain how exactly information and evaluations derived visually and verbally are combined. Also one wonders what is behind the dispositional preferences for visualizers and verbalizers in the first place and whether it is possible to change a visualizer into a verbalizer or vice versa in terms of dispositions.

A final contribution by Wyer et al. (in press) that can be mentioned concerns their clever studies of narrative-based processing. The reader is referred to their article, as well as Wyer and Radvansky (1999) and Wyer (2004) for background and other research. Only brief comments will be made here on a limited set of this research. In the Hung and Wyer (2007) study, where both problem and solution elements are presented verbally, respondents paid little attention to ads and failed to construe intended implications. In this case, one wonders whether the ads were poorly constructed and whether creatives at an ad agency could overcome attentional and comprehension problem. More generally, it would be interesting to study what design aspects of ads facilitate information processing and what elements might even overcome the effects found by Hung and Wyer (2007). The descriptions provided by Wyer et al. (in press) concerning the Hung and Wyer (2007) study were very confusing to this reader. This is one case where the original article will be needed to be consulted to comprehend the theory and findings fully.

\section{Style-of-processing: interesting idea, poor measures}

Researchers sometimes use scales without demonstrating the psychometric properties of the items in the scale for their particular application at hand (e.g., dimensionality, reliability, validity). Occasionally, the scales that researchers use have not been validated much psychometrically or have been misinterpreted or misrepresented by the originators of the scales. In both cases, it is tempting to overlook such outcomes or practices, when the use of the scale seemingly accomplishes its purpose in a study at hand. The rationale seems to be, if it works as intended, then it must be reliable and valid, and the findings based on the use of the scale must be meaningful and interpretable. This conclusion is quite fallacious. Invalid or poor scales can yield seemingly interpretable results for the wrong reasons or for unknown reasons. It is important to rely on valid scales and to demonstrate key psychometric properties of the measures of the scale in any application of it, before and in conjunction with interpreting findings based upon it.

A number of studies summarized by Wyer et al. (in press) use the Style-of-Processing (SOP) scale to operationalize chronic individual differences in the disposition to process information verbally or visually (e.g., Jiang et al., 2007; Jiang \& Wyer, 2007). The SOP scale is a 22-item, 4-point true-false scale, with 11 items for measuring verbal, and 11 items for measuring visual, dispositions to process information semantically or to construct visual images, respectively, when people are engaged in different mental tasks (see Childers, Houston, \& Heckler, 1985).

In the original development of the scale (Childers et al., 1985, pp. 130-132), 54 undergraduates filled-out the SOP scale, and LISREL was applied to test a two-factor visual-verbal confirmatory factor analysis model. The only information provided as to model goodness-of-fit was $\chi^{2}(208)=464.72$, $p<.001$. The normal interpretation of this goodness-of-fit result should have led the authors to reject the model, as a nonsignificant $\chi^{2}$ is desired (i.e., $p>.05$ ). Nowadays, researchers do not rely solely on the $\chi^{2}$-test because of its sensitivity to sample size and other shortcomings (e.g., Hu \& Bentler, 1998, 1999). A common concern is that the sensitivity of the $\chi^{2}$-test to sample size will often lead to rejection of good models (i.e., the larger the sample size, the higher the $\chi^{2}$ value). But in the original study conducted by Childers et al. (1985), the sample size $(N=54)$ is considerably smaller than generally desired, which means that the danger exists that one could, on the basis of the value of the $\chi^{2}$-test, accept a poor model. This means that the poorly fitting model found by Childers et al. is actually more disconcerting than meets the eye. The proper interpretation of the findings presented in Childers et al. (1985) is that the proposed model does not fit the data and should be rejected. This means that even convergent validity of the multiple measures of each component (the verbal and visual) has to be rejected (on the basis of the interpretation of the $\chi^{2}$-test). Given this, the tests and interpretations of discriminant validity and criterion validity presented in their article are not meaningful (i.e., convergent validity must be established before examining discriminate or other types of validity).

There is another issue to mention. Childers et al. tested an orthogonal model, assuming that verbal and visual dispositions are perfectly uncorrelated. Had the factors corresponding to the two dispositions been significantly correlated in reality, even at 
a low level, this could have contributed to the poorly fitting model that they found. It would have been better to also run an oblique model and explicitly test for the significance of the correlation between dispositions (by use of a $\chi^{2}$ difference test for example).

This raises the question of whether the dispositions to process information semantically and to construct visual images are mutually exclusive tendencies, as assumed by Childers et al. The items proposed by Childers et al. for the SOP scale are of three kinds: beliefs about statements of fact (e.g., "I spend very little time attempting to increase my vocabulary"), affect or ongoing desires (e.g., "I enjoy doing work that requires the use of words"), and evaluations or preferences (e.g., "I prefer activities that don't require a lot of reading", "I like to 'doodle""). Because the authors defined processing style to be "a preference and propensity to engage in a verbal and/or visual modality of processing" (Childers et al., 1985, p. 130), the items on the scale constitute direct and indirect measures of the concepts behind the scale. It would be better to rely only on more direct measures of preference or propensity and to avoid affective ones; statements of fact concerning actions that one does verbally or visually might be $\mathrm{OK}$ to use too, but to the extent that respondents do such actions, yet without a preference or propensity for doing so (e.g., because they are required to do so or do so out of a habit acquired out of duty or force), such statements of fact may introduce systematic error. In any case, it seems possible that at least some people do, or have a propensity for, both verbal and visual processing, and therefore would respond similarly to such items on the SOP scale as "I do a lot of reading", "My thinking often consists of mental 'pictures' or images", "I enjoy learning new words", and "I like to daydream", which allegedly measure either verbal or visual propensities for processing. It should be noted, too, that verbal and visual propensities are sometimes built into the same item on the SOP scale, where the respondent is asked to make a choice based on a comparison of the two modes of processing (e.g., "When I'm trying to learn something new, I'd rather watch a demonstration than read how to do it"), while many items have only verbal or visual propensities as referents. This practice also likely introduces some error in responses. Finally, instead of the 4-point "always true/always false" scale, it would be better to use at least 5-point scales where, for example, respondents indicate to what extent items pertain to them (e.g., "Does not describe me at all" to "Describes me completely", with "Describes me moderately well" in between). Five-point scales and higher, but not 4-point scales, have been shown to lead to satisfactory distributional properties of observations for confirmatory factor analysis models estimated with maximum likelihood estimation (Olsson, 1979), and a "does not describedescribes me completely" scale is a more meaningful measure of disposition possession (because it can be adapted to measure extent or magnitude of a disposition) than an "always truealways false" scale (which might be ambiguous because it reflects frequency of exhibiting a disposition, not its intensity).

Perhaps the above ambiguities in the SOP scale account for its poor performance in terms of model fit. In any case, the findings for the LISREL estimates reported by Childers et al.
(1985, Table 3), which they assert "tend to support the structure and consistency of the SOP scale" (p. 131), indicate serious problems with the scale. At least 4 of 11 items for the visual component (items 5, 11, 12, and 14) and 4 of 11 items for the verbal component (items 3, 6, 9, and 18) point to poor convergent validity, with 4 additional visual items (items 2,8 , 10, and 20) and 6 additional verbal items (items 1, 4, 7, 15, 17, and 19) suggesting mediocre convergent validity. In addition, the wide-range in loadings ( .39 to .87 for the visual component, and .22 to .77 for the verbal component) imply that neither the visual nor the verbal component may be unidimensional, or alternatively, at a minimum some items measure something other than propensity to engage in verbal or visual processing. Either outcome brings into question the validity of the scale.

The above observations suggest that a need and opportunity exist for developing a valid scale to measure chronic processing styles. In the meantime, it may be best to manipulate processing styles by priming or other means, if one wishes to investigate the effects of visual and verbal processing strategies. The possibility that people may be inclined to use both orientations could also be conceptualized more clearly and measured appropriately.

\section{Philosophical and neuroscience reflections}

The research reported in Wyer et al. (in press) is informed by Wyer's (2004) information processing system, which Wyer and Radvansky (1999, p.92) characterize as a "metaphorical representation". As mentioned above, the nature of the connection between the studies discussed in Wyer et al. (in press) and the information processing system has not been developed in much detail. The authors seem to treat the information system more as a heuristic than as a model or theory that is tested in relatively holistic, as opposed to piece-meal, ways. Indeed, it is possible to interpret the studies by Wyer et al. without recourse to the framework, as the studies can stand alone. The question remains whether a more formal theory can be developed that deepens and makes explicit the information processing system such that existing and future research can be shown to be interpreted within it and in a way that new predictions can be made. Other areas in the social sciences place more emphasis on the formal specification of theoretical frameworks and their explicit relationship to observations or measurements. In these areas, larger models are tested, or at least relatively large parts of models are investigated, so as to yield one or few paradigmatic frameworks. But in psychology, the tradition is to test many very circumspect and well defined hypotheses each of which is generally loosely tied to a larger framework, and the framework itself is rarely addressed directly. The result is a large number of studies that give the impression of fragmentation in the field, in comparison to other areas in the social sciences for which the linkage between individual studies and an organizing theory(ies) is made more explicit. One wonders whether more effort at middle-range theorizing would bring the disparate findings across studies in psychology together and in so doing more clearly point to a small number of integrated directions for future study. To do this 
may require introducing other methodologies to complement the heretofore exclusive reliance on experimentation.

In addition to the nature of the information system and its specificity and its relationship to the body of current research, one might ask how the processes summarized in the findings in Wyer et al. (in press) are realized in the brain. How do neural processes corresponding to the findings cause or produce comprehension and judgment? What are the logical structures of comprehension and judgment and how do these relate to the findings in Wyer et al. (in press) and brain processes? Can these be expressed in a first-person ontology where the subjective experiences of people are defining? This question is especially important to address when we do research into human judgment and decision making with respect to specific actions, where people take into account beliefs, feelings, etc. as personal reasons for acting or not. Yet much of research into human memory and decision making seems to rest on third-person conceptions and assumptions, and views the effects of comprehension on judgment, the effects of judgment on decisions, and the effects of decisions on volitions and action as not under deliberative or self-regulatory control, per se. How do cognitive processes and our use of language commit us to act or refrain from acting? How are memory, beliefs, comprehension, etc. represented in language and how is language derived from them? It would seem too that language can be an expression of comprehension, judgment, etc.

One leading view in neuroscience seems to claim that comprehension, judgment, decision making, planning, etc. are determined or caused by brain processes such as neuronal firings. If so, can these brain processes be formally linked to comprehensions, etc.? In what sense might comprehension, etc. be qualitative and subjective, and how can we represent and study these processes and relate these to brain activity?

Recently a number of challenges to memory research have been raised by neuroscientists (e.g., Bennett \& Hacker, 2003). I do not personally come from the same school of thought that Bennett and Hacker (2003) do, and I disagree with many of their claims, but some of the questions they raise can help researchers studying memory and information processing better relate their hypotheses and findings to brain processes. For example, Bennett and Hacker (2003, p. 29) assert that much confusion in neuroscience and psychology occurs because of a mereological fallacy, which they define as "ascribing to a part of a creature attributes which logically can be ascribed only to the creature as a whole". Thus they claim that it is wrong to ascribe psychological attributes to the brain (e.g., to believe that the brain sees, hears, has experiences), but rather it is the human person who sees, hears, etc. They go farther by claiming that we perceive physical things and that these result in physical events and processes in the brain, but we do not perceive or process images or representations. All there is in the brain, they claim, are neurons firing, but no information per se and no images, visual or otherwise. Wyer et al. (in press, fn 1) mention how useful and "incontrovertible" visual image constructs are in conceptualizing comprehension and judgment. If we are to show how such images exist and are produced by brain processes, a theory will need to be developed showing how images are linked to comprehension and judgment and how all these are realized in brain activity. Likewise the meaning and representation of knowledge, situation models, generalized representations, and other concepts and operations depicted in the information system will also need to be specified more fully if they are to be shown to be caused by or realized in brain activity. Researchers do not have to model comprehension and judgment in brain processes to achieve insights of the sort Wyer et al. (in press) attain, and the methods used by Wyer et al. (in press) will continue to have much currency. But there may be some discoveries that are best approached with neuroscience or that can inform the type of experimental work and metaphors with which Wyer and other psychologists deal.

\section{Final thoughts}

Professor Wyer and colleagues have made many fundamental contributions to social comprehension and judgment. This occurs at the level of theory and their original conceptual framework for thinking about information processing (e.g., Wyer, 2004; Wyer \& Radvansky, 1999; Wyer \& Srull, 1989), and at the level of empirical research, for which Wyer et al. (in press) present a comprehensive body of work on processing of information in different sense modalities and relate it to Wyer's conceptual framework.

A number of additional directions for research in social comprehension and judgment come to mind. For example, in both the processes of comprehension and goal-setting and goalstriving, one wonders how more active processing might occur in persuasive communication contexts as a result of such cognitive responses as counter argumentation, support argumentation, source derogation, and source bolstering. In Wyer's (2004, p. 30) model, it appears that cognitive responses of the type mentioned occur in the response selector and executor parts of the architecture and relate to goal-directed processes, but I am unaware of explicit consideration of such responses and empirical work with respect to social judgment differences in verbal and visual modalities and preferences. This would seem to be a fruitful direction for new research. Likewise cognitive responses may even play a role in what Wyer terms, the comprehender. The comprehender is an initial encoding mechanism that operates automatically to interpret new information. It is believed to draw upon declarative knowledge in permanent memory. Can the automaticity of the comprehender be subject to influence by cognitive responses, and might this differ between verbal and visual modes of processing styles? Wyer's model has a path specified from permanent storage to the comprehender in his framework (Wyer, 2004), but not from the work space to the comprehender, and thus it is unclear how or whether cognitive responses can shape comprehension according to his theory.

Still another question concerns the role of affect in social comprehension and judgment in relation to different modalities. Wyer (2004, p.9) maintains that affect is not part of the cognitive system, but affective reactions, as "conditioned or unconditioned responses to cognitions", function sometimes as "referents of cognitions". Because emotions are distinct from 
verbal-semantic processes, yet can be conditioned to words and things we see or sense, they potentially can affect comprehension and decision making. How this is done seems in need of more theorizing. Related to this is the question of how affect relates to goal setting, goal choice, and goal pursuit. Affect seems essential in decision making involving a commitment to a course of action, but where this fits in Wyer's model is unclear. More generally, how does affect influence comprehension, judgment, choice, and decision making when information is received in different modalities and where does motivation fit in the information processing system?

A related opportunity concerns sensory processes and their role in social comprehension, judgment, and decision making. The relationship between memory and odors, for example, can be stronger than that between memory and our other senses, and may even differ in kind from these (Herz, 1998). Scents profoundly affect how we comprehend, think about, and make decisions about ourselves and others, as well as consumption. Some of these effects arise through learned associations with emotions on initial exposure to particular odors, and there are differential responses to the same scents not only crossculturally but within cultures. In any case, odors influence social comprehension and goal-directed processes and are intimately related to interpersonal and individual consumption behaviors (e.g., Milinski \& Wedekind, 2001; Spangenberg, Sprott, Grohmann, \& Tracy, 2006). How odors, in particular, and sensory processes, in general, function in comprehension and decision making, when information is presented in visual, verbal, olfactory, and other sensory modes, are fruitful areas for future research. Unlike our other senses where responses are innate, olfactory responses entail strong learning characteristics and therefore show unique and variable opportunities for social comprehension and goal-directed processes.

A final area of enquiry worth considering is the role of social comprehension and higher cognitive processes in goal striving. Much of research to date follows a model or sequence of comprehension/information processing $\rightarrow$ decision making $\rightarrow$ behavior, where cognitive processes seemingly stop with decision making. But what are the roles of comprehension and higher cognitive processes in post-decision making activities, and do visual and verbal strategies and dispositions have consequences here? For example, Gollwitzer's notion of implementation intentions, where the planning of when, where, and how to act so as to achieve a goal takes place, rests on the future activation of cues occurring immediately prior to an opportunity for acting so as to trigger volitions and motivation needed in goal pursuit and for goal achievement. The learning of visual and verbal information and differential cueing of learned visual and verbal information, after planning takes place, might affect how well implementation intentions work. Indeed there are many different kinds of cognitive, motivational, and affective processes that might occur after decision making, but before goal attainment, and even before action taken to achieve a goal, which Wyer's information processing system and the research reported in Wyer et al. (in press) might have much to say. An unresolved, related issue is how exactly do comprehension and higher cognitive processes produce or induce actual behavior? Behavior is apparently a consequence of the operation of Wyer's information processions system, but little is said in this regard in his theorizing, which represents still another avenue for future research.

\section{References}

Alba, J. W., Hutchinson, J. W., \& Lynch, J. G., Jr. (1991). Memory and decision making. In T. S. Robertson, \& H. H. Kassarjian (Eds.), Handbook of consumer Research (pp. 1-49). Prentice Hall: Englewood cliffs, NJ.

Barsalou, L. W. (1991). Deriving categories to achieve goals. In G. H. Bower (Ed.), The Psychology of Learning and Motivation, Vol. 27. (pp. 1-64) New York: Academic Press.

Bennett, M. R., \& Hacker, P. M. S. (2003). Philosophical Foundations of Neuroscience. Malden, MA: Blackwell.

Childers, T. L., Houston, M. J., \& Heckler, S. E. (1985). Measurement of individual differences in visual versus verbal information processing. Journal of Consumer Research, 12, 125-134.

Escalas, J. E., \& Luce, M. F. (2004). Understanding the effects of processfocused versus outcome-focused thought in response to advertising. Journal of Consumer Research, 31, 274-285.

Herz, R. S. (1998). Are odors the best cues to memory? A cross-modal comparison of associative memory stimuli. Annals of the New York Academy of Sciences, 855, 670-674.

Hu, L., \& Bentler, P. M. (1998). Fit indices in covariance structure modeling: sensitivity to underparameterized model misspecification. Psychological Methods, 3, 424-453.

Hu, L., \& Bentler, P. M. (1999). Cutoff criteria for fit indexes in covariance structure analysis: conventional criteria versus new alternatives. Structural Equation Modeling, 6, 1-55.

Hung, I.W., \& Wyer, R.S. Jr. (2007). The impact of implicit theories on responses to problem-solving print advertisements. Unpublished manuscripts, Hong Kong University of Science and Technology.

Jiang, Y., Steinhart, Y. \& Wyer, R.S. Jr. (2007). The role of visual and semantic processing strategies in consumer information processing. Unpublished manuscript, Hong Kong University of Science and Technology.

Jiang, Y., \& Wyer, R.S., Jr. (2007). The role of visual perspective in information processing. Unublished manuscript, Hong Kong University of Science and Technology.

Luce, M. F. (1998). Choosing to avoid: coping with negatively emotion-laden consumer decisions. Journal of Consumer Research, 24, 409-433.

Milinski, M., \& Wedekind, C. (2001). Evidence for MHC-correlated perfume preferences in humans. Behavioral Ecology, 12, 140-149.

Olsson, U. (1979). On the robustmess of factor analysis against crude classification of observation. Multivariate Behavioral Research, 14, 485-500.

Schwarz, N. (2004). Metacognitive experiences in consumer judgment and decision making. Journal of Consumer Psychology, 14, 332-348.

Spangenberg, E. R., Sprott, D. E., Grohmann, B., \& Tracy, D. L. (2006) Gender-congruent ambient scent influences on approach and avoidance behaviors in a retail store. Journal of Business Research, 59, 1281-1287.

Unnava, H. R., \& Burnkrant, R. E. (1991). An imagery-processing view of the role of pictures in print advertisements. Journal of Marketing Research, 28, 226-231.

Wyer, R. S. (2004). Social comprehension and judgment: The role of situation models, narratives, and implicit theories. Mahwah, NJ: Erlbaum.

Wyer, R. S., \& Srull, T. K. (1989). Memory and cognition in its social context. Hillsdale, NJ: Lawrence Erlbaum Associates, Inc.

Wyer, R. S., Jr., Adval, R., \& Colcombe, S. J. (2002). Narrative-based representations of social knowledge: their construction and use in comprehension, memory and judgment. In M. P. Zanna (Ed.), Advance in experimental social psychology, vol. 34. (pp. 131-197) San Diego, CA: Academic Press.

Wyer, R.S. Jr., Hung, I.W., \& Jiang, Y. (in press). Visual and verbal processing strategies in comprehension and judgment, Journal of Consumer Psychology.

Wyer, R. S., Jr., \& Radvansky, G. A. (1999). The comprehension and validation of social information. Psychological Review, 106, 89-118. 